\title{
A randomized controlled efficacy trial of an electronic screening and brief intervention for alcohol misuse in adolescents and young adults vulnerable to HIV infection: step up, test up study protocol
}

Lisa M. Kuhns ${ }^{1,2^{*}}$, Niranjan Karnik ${ }^{3}$, Anna Hotton $^{4}$, Abigail Muldoon $^{1}$, Geri Donenberg ${ }^{5}$, Kristin Keglovitz $^{6}$, Moira McNulty ${ }^{4}$, John Schneider ${ }^{4,6}$, Faith Summersett-Williams ${ }^{1,7}$ and Robert Garofalo ${ }^{1,2}$

\begin{abstract}
Background: Young people account for more than a quarter of new HIV infections in the US, with the majority of cases among young men who have sex with men; young transgender women are also vulnerable to infection. Substance use, particularly alcohol misuse, is a driver of sexual transmission and a potential barrier to engagement in the HIV prevention and care continuum, however vulnerable youth are difficult to reach for substance use services due, in part, to complex social and structural factors and limited access to health care. The Community Prevention Services Task Force recommends electronic screening and brief intervention as an evidence-based intervention for the prevention of excessive alcohol consumption; however, no prior studies have extended this model to community-based populations of youth that are susceptible to HIV infection. This paper describes the study protocol for an electronic screening and brief intervention to reduce alcohol misuse among adolescents and young adults vulnerable to HIV infection in community-based settings.
\end{abstract}

Methods: This study, Step Up, Test Up, is a randomized controlled trial of an electronic alcohol screening and brief intervention among youth, ages 16-25, who are vulnerable to HIV infection. Individuals who present for HIV testing at one of three community-based locations are recruited for study participation. Eligibility includes those aged 1625 years, HIV-negative or unknown HIV status, male or trans female with a history of sex with men, and Englishspeaking. Participants who screen at moderate to high risk for alcohol misuse on the Alcohol Use Disorders Identification Test (AUDIT) are randomized (1:1) to either an electronic brief intervention to reduce alcohol misuse or a time-and attention-matched control. The primary outcome is change in the frequency/quantity of recent alcohol use at 1, 3, 6 and 12-month follow-up.

Discussion: Testing of evidence-based interventions to reduce alcohol misuse among youth vulnerable to HIV infection are needed. This study will provide evidence to determine feasibility and efficacy of a brief electronicallydelivered intervention to reduce alcohol misuse for this population.

Trial registration: ClinicalTrials.gov number, NCT02703116, registered March 9, 2016.

Keywords: HIV prevention, Men who have sex with men, Transgender women, Alcohol intervention

\footnotetext{
* Correspondence: Ikuhns@luriechildrens.org

'The Potocsnak Family Division of Adolescent and Young Adult Medicine,

Ann \& Robert H. Lurie Children's Hospital, 225 E. Chicago Avenue, Box 161,

Chicago, IL 60611, USA

${ }^{2}$ Department of Pediatrics, Northwestern University, Feinberg School of

Medicine, Chicago, IL, USA

Full list of author information is available at the end of the article
}

(c) The Author(s). 2020 Open Access This article is distributed under the terms of the Creative Commons Attribution 4.0 International License (http://creativecommons.org/licenses/by/4.0/), which permits unrestricted use, distribution, and reproduction in any medium, provided you give appropriate credit to the original author(s) and the source, provide a link to the Creative Commons license, and indicate if changes were made. The Creative Commons Public Domain Dedication waiver (http://creativecommons.org/publicdomain/zero/1.0/) applies to the data made available in this article, unless otherwise stated. 


\section{Background}

Recent estimates of U.S. HIV incidence indicate that young people aged 13 to 24 account for nearly a quarter of new infections, with the majority of cases among young men who have sex with men (YMSM) [1, 2]. While official incidence statistics are not differentiated by transgender status, local estimates indicate extremely high rates of unrecognized HIV infection among young transgender women (YTW) as well [3-5]. Substance use, particularly alcohol misuse, has been identified as a driver of sexual transmission of HIV infection [6-9] and a potential barrier to engagement in HIV prevention and care among YMSM and YTW, however these youth are difficult to reach for substance use services due, in part, to complex social and structural factors and limited access to health care. YMSM and YTW, however, often seek HIV testing and other supportive services in community-based and outreach settings. These settings are underutilized as potential entry points for engagement in comprehensive care across the HIV prevention and care continuum, including pre-exposure prophylaxis (PrEP) for HIV-negative youth who are at risk of HIV acquisition.

Given the barriers to substance use screening and intervention among YMSM and YTW, interventions are needed that are client-centered and can be deployed in community settings. Motivational interviewing (MI) is a well-established client-centered behavioral change approach $[10,11]$. The theoretical basis for MI draws in part on Rogerian concepts of psychotherapy that emphasize the need for self-exploration and patientcenteredness. The core of MI emphasizes that individuals must be empowered to change and that given the right set of circumstances they will gravitate toward more pro-social outcomes. The hallmarks of this method avoid telling individuals what to do, and instead create a space where the individual is allowed to explore their motivations through discrepancies that exist in their life (i.e. alcohol makes me feel relaxed, but I don't like the way I treat others when I drink). By seeing these discrepancies, individual find a motivation to change and then move forward.

Expansion of MI-based or MI-informed approaches is taking place via initiatives to promote screening, brief intervention and referral to treatment (aka SBIRT). Electronic screening and brief intervention (eSBI) is a subset of SBIRT and essentially takes SBIRT into an electronic medium suitable for use in primary care and other generalist settings. The Community Prevention Services Task Force (CPSTF) recommends eSBI as an evidencebased intervention for the prevention of excessive alcohol consumption [12]. Most studies to date have tested this approach in health care (e.g., emergency room) and university settings with adults and young adults and for relatively short follow-up time (i.e., 6-months post intervention). In these settings, the CPSTF found sufficient evidence of efficacy across studies and age groups [12]. Little trial data exists for the use of eSBI in adolescents, minority racial/ethnic groups, or for longer periods of follow-up. To date no studies have tried to extend this model to populations such as YMSM and YTW, in community-based settings in which these youth tend to access services.

To capitalize on this opportunity, we propose to test a structural change to the HIV prevention and care continuum by integrating substance use screening and brief intervention into the traditional community-based HIV testing environment. We will couple standard HIV prevention with $\mathrm{eSBI}$ for at the point of HIV testing.

\section{Study objectives}

The purpose of this study is to assess the feasibility, acceptability, and initial efficacy of eSBI in comparison to an electronic attention control intervention (i.e., promotion of good nutrition), coupled with standard HIV prevention, on alcohol use among YMSM and YTW in community-based HIV testing environments in Chicago. Secondary objectives are to assess intervention effects on sexual behavior, as well as engagement within the HIV prevention and care continuum, and to assess modification of the intervention effect by co-morbid mental health problems (i.e., symptoms of depression, anxiety, and exposure to trauma).

\section{Methods/design \\ Design}

This study is an individually randomized controlled trial (RCT) of eSBI among youth vulnerable to HIV infection. Using an electronic portal, all participants are screened for alcohol misuse and receive immediate feedback regarding their level of use (e.g., how their use compares to others, whether it exceeds "safe use" guidelines); those who screen for moderate to high alcohol use, including binge drinking, are then randomized to either electronic intervention or control modules. All participants, regardless of randomization status, are followed for 12 months with in-person visits conducted at 1, 3, 6 and 12-month intervals (Fig. 1).

\section{Identification and recruitment of participants}

Youth are recruited from HIV testing centers at the three primary sites in Chicago: the Division of Adolescent Medicine at Lurie Children's Hospital, Howard Brown Health, and the Village at the University of Chicago. Individuals are eligible if they are: (a) aged 16 to 25 years, (b) interested in testing for HIV infection and (c) HIV-negative or HIV status unknown (per selfreport; verified at the point of HIV testing), and (d) are 


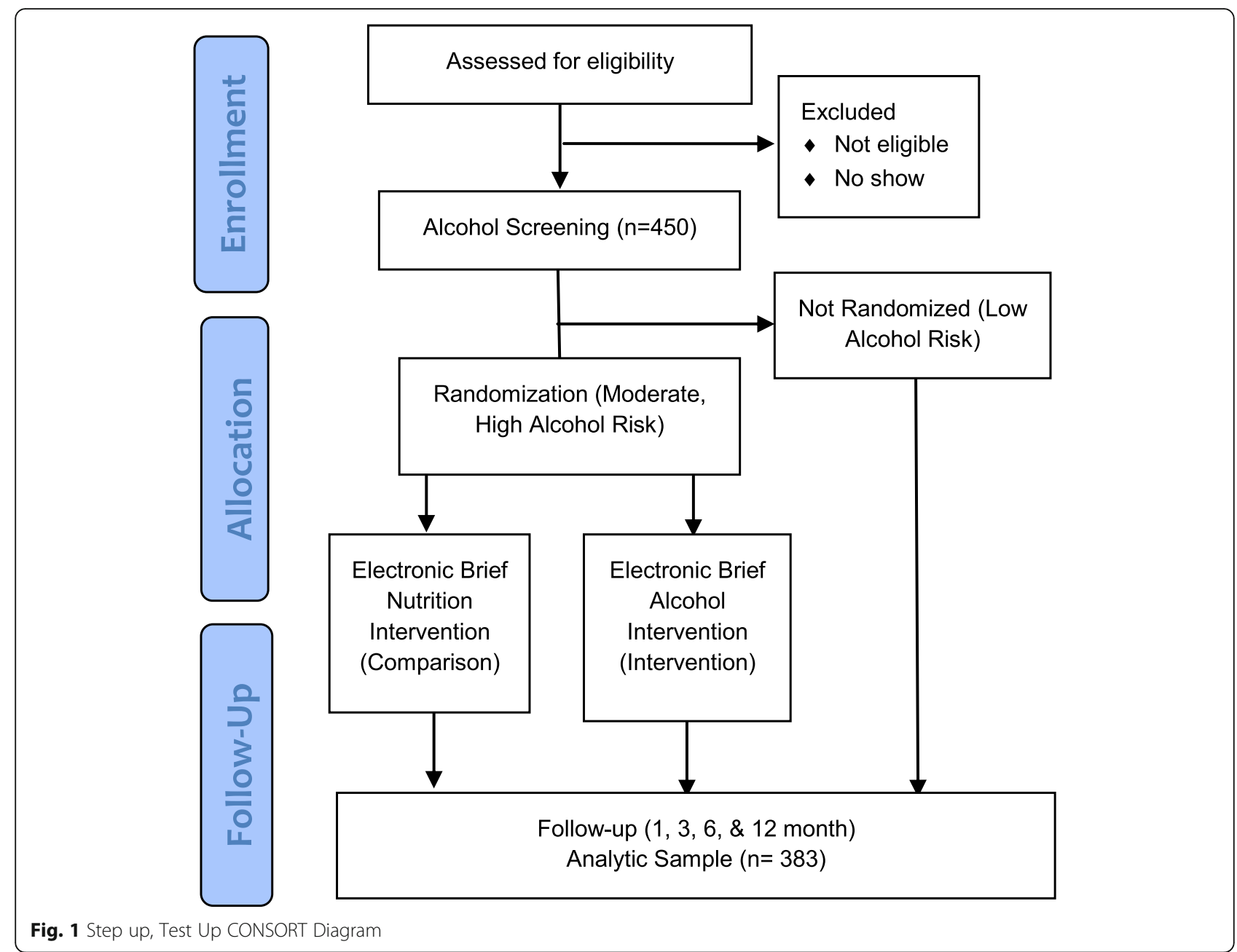

MSM or a transgender woman who has sex with men (i.e., born male, identify as female/transgender, and at any point in the gender transition process); (e) Englishspeaking.

\section{Randomization}

Upon enrollment, all participants complete HIV testing and screen for alcohol misuse using the Alcohol Use Disorders Identification Test (AUDIT) via an electronic portal, then receive immediate feedback regarding their level of use. Participants who screen moderate to high risk for alcohol misuse or endorse binge drinking on the AUDIT are randomized via computerized assignment in the portal (1:1) to either brief intervention modules to reduce alcohol misuse or a time-and attention-matched control (i.e., promotion of good nutrition). Study staff are blinded to random assignments.

\section{Description of the intervention: eSBI}

Those randomized to the intervention complete modules on 11 topical areas (see Table 1), each with a single webpage, in an MI format. The intervention content was targeted to late adolescents and young adults in terms of voice and language (e.g., open, non-authoritarian, inclusive), contexts (e.g., school as well as work contexts) and reading level. Completion of the intervention is expected

Table 1 eSBI Topical Areas

\begin{tabular}{ll}
\hline Webpage & Topic \\
\hline 1 & Importance of change \\
2 & Downsides of drinking \\
3 & Confidence scale \\
4 & Barriers to change \\
5 & Explore ways to cut back or quit \\
6 & Strategies for cutting back \\
7 & Change motivators \\
8 & 1 year from now \\
9 & Readiness to change \\
10 & Summary slide \\
11 & More information and/or referral \\
\hline
\end{tabular}


to take 10-15 min. The electronic intervention and control modules are delivered using a customized eSBI platform developed by Radiant Interactive Group (Laguna Niguel, CA). Radiant uses industry-standard data encryption and security to manage Protected Health Information recorded by their system.

\section{Time- and attention-matched control: diet and nutrition} Those randomized to control complete a brief timematched attention control intervention, also in MI format, of equal length, which encourages good nutrition.

\section{Standard-of-care prevention services}

The standard HIV preventive care at each site includes youth-specific HIV testing and counseling and linkage navigation to PrEP or HIV care.

\section{Study assessments}

Participants complete a baseline study visit comprised of standardized assessment via computer-assisted selfinterviewing (CASI). Follow-up CASI assessments are conducted at 1, 3, 6, and 12-month follow-ups visits. Detailed contact information is collected in order to facilitate study retention.

Primary Outcome: Change in the frequency/quantity of self-reported recent alcohol use at 1, 3, 6 and 12months on the Daily Drinking Questionnaire [13].

Secondary Outcomes: Sexual risk behavior is measured via a modified version of the AIDS-Risk Behavior Assessment [14] used in prior studies of YMSM and YTW $[15,16]$. Engagement in HIV and/or PrEP care is measured via medical records abstraction of clinic visit continuity (i.e., at least two clinic visits over the 12-month observation period).

Moderators: Depression, anxiety, and trauma will be measured using the short version of the Center for Epidemiologic Studies Depression Scale (CES-D10), [17] 7item Generalized Anxiety Disorder (GAD-7) [18] and Life Events Checklist (LEC), [19] respectively.

\section{Statistical analysis}

Several data quality checks will be conducted prior to analysis, including analysis of descriptive statistics and graphic plots to detect appropriate range of variables and to detect missing data or invalid cases. All data are stored in a password-protected network server with daily back-up. In order to assess the initial efficacy of eSBI, the intervention and control groups will be compared on baseline characteristics to assess randomization, and variables for which there are significant group differences will be controlled in subsequent analyses. Extensions of generalized linear models (GLMs) will be used to assess the effect of the intervention on the primary alcoholrelated outcomes at 1, 3, 6 and 12-month follow-up visits (post-intervention). Intervention effects will be examined in terms of average group differences over follow-up and using models with an indicator for intervention group, time, and a group by time interaction to determine whether the intervention and control groups differ in response to the intervention over time. Potential confounding and effect modification by sociodemographic characteristics and co-morbid conditions will be examined in exploratory analysis, and these variables will be selected for inclusion in multivariable models based on statistical significance and conceptual importance. All models will account for correlation among repeated measures on individuals over time as appropriate (e.g., by inclusion of subject-level random effects). Potential modifiers of the intervention effect on outcomes will be examined using models including two-and three-way interactions of intervention group, time, and moderator effects to determine whether intervention effects differ across levels of the modifying variables.

\section{Sample size calculation}

We propose to enroll 450 participants and have estimated $80 \%$ power to detect effects for our primary (alcohol use) and secondary (PrEP uptake) outcomes using formulas for longitudinal study designs with attrition [20]. Calculations assume an exchangeable covariance structure, measurements at baseline and 4 follow up points, and within-subject correlation among repeated measurements (rho) of 0.5. All estimates use two-sided tests of significance and alpha of 0.05 . With a retention rate of $85 \%$ at 1 year, we would have $>80 \%$ power to detect effect sizes (Cohen's d) of 0.23 (small effect size) [21] or greater for between-group comparison of continuous measures of alcohol consumption and other substance use. For binary outcomes (i.e. any binge drinking), we would have $\geq 80 \%$ power to detect differences in proportions of $10 \%$ or greater when the proportion in the control group ranges from 25 to $30 \%$, using a repeated measures framework with 4 follow-up measures and assumptions stated above. Thus, this study is powered to detect small to medium effect sizes that are consistent with or smaller than others reported in the literature [22].

\section{Discussion}

We describe herein the design of a randomized controlled trial of an eSBI, "Step Up, Test Up," to reduce alcohol misuse among youth, ages 16-25 who are vulnerable to HIV infection in Chicago. The intervention draws on MIinformed approaches, including SBIRT, adapted in an electronic medium suitable for use in primary care and other generalist settings. We hope to extend the evidence base for eSBI to a group vulnerable to alcohol misuse, but with limited opportunity for intervention in traditional 
settings. We believe the design of this study has several strengths, including its focus on a younger group vulnerable to both alcohol misuse and related consequences, using a randomized controlled design in communitybased practice environments where youth often seek testing for HIV infection. Furthermore, we believe that this intervention is developmentally responsive and addresses the age range with the steepest rise in both alcohol misuse and HIV acquisition (i.e, ages $16-25$ years).

Step Up, Test Up, is among the first interventions to be tested in a rigorous trial of sufficient size to detect effects of eSBI and targeting youth who are vulnerable to alcohol misuse and HIV infection. We consider it a potential innovation in the HIV testing environment, which given the brief and electronic format, is well positioned for scale. It was designed to meet the standard for evidence-based interventions and to extend the base of evidence for intervention with this population with many assets, yet increased susceptibility to HIV and other STIs.

\section{Abbreviations}

ARBA: AIDS Risk Behavior Assessment; AUDIT: Alcohol Use Disorders Identification Test; CASI: Computer Assisted Self Interviewing; CDC: Centers for Disease Control and Prevention; CES-D10: Center for Epidemiological Studies Depression 10-item Scale; CPSTF: Community Prevention Services Task Force; eSBI: Electronic Screening and Brief Intervention; GAD7: Generalized Anxiety Disorder 7-item Scale; GEE: Generalized Estimating Equations; GLM: Generalized Linear Models; HIV: Human Immunodeficiency Virus; LEC: Life Events Checklist; MI: Motivational Interviewing; NIH: National Institutes of Health; PrEP: Pre-Exposure Prophylaxis; RCT: Randomized Controlled Trial; SBI: Screening \& Brief Intervention; SBIRT: Screening, Brief Intervention \& Referral to Treatment; US: United States; YMSM: Young Men Who Have Sex with Men; YTW: Young Transgender Women
\end{abstract}

\section{Acknowledgements}

We would like to thank member of the Step Up, Test Up team at all study sites for their contribution to study protocol and procedures.

\section{Authors' contributions}

LK, NK and RG contributed to the design of all aspects of the study. GD, JS, $\mathrm{KK}$ and MM contributed to the study design and selection of measures. $\mathrm{AH}$ designed the proposed data analysis. AM created Fig. 1. LK and NK drafted the manuscript. The manuscript has been read and approved by all authors.

\section{Funding}

This study is funded by the National Institute on Drug Abuse of the National Institutes of Health under Award Number R01DA041071. The content is solely the responsibility of the authors and does not necessarily represent the official views of the $\mathrm{NIH}$.

\section{Availability of data and materials}

Not applicable.

\section{Ethics approval and consent to participate}

This protocol has been approved by the Institutional Review Boards of Ann \& Robert H. Lurie Children's Hospital of Chicago (IRB 2011-14600), Rush University (IRB 15040605-IRB01), University of Chicago, (IRB IRB15-1454) and the University of Illinois at Chicago (IRB\#2016-0190) respectively, with a waiver of parental permission for participation of minors (aged 16-17). The IRB of Howard Brown Health deferred to the Lurie Children's IRB for this protocol. Study participants complete an informed consent process prior to participation in research activities through a web portal with a waiver of written consent. This study is monitored by a Data Safety and Monitoring Board (DSMB), the composition of which may be obtained from the study principal investigator at the lead institution. No criteria for discontinuing or modifying the intervention or trial stopping rules were defined for this study.

\section{Consent for publication}

Not applicable.

\section{Competing interests}

The authors declare that they have no competing interests.

\section{Author details}

${ }^{1}$ The Potocsnak Family Division of Adolescent and Young Adult Medicine, Ann \& Robert H. Lurie Children's Hospital, 225 E. Chicago Avenue, Box 161, Chicago, IL 60611, USA. ${ }^{2}$ Department of Pediatrics, Northwestern University, Feinberg School of Medicine, Chicago, IL, USA. ${ }^{3}$ Department of Psychiatry \& Behavioral Sciences, Rush Medical College, Rush University, Chicago, IL, USA. ${ }^{4}$ Chicago Center for HIV Elimination, University of Chicago Medicine,

Chicago, IL, USA. ${ }^{5}$ Department of Medicine, University of Illinois at Chicago, Center for Dissemination and Implementation Science, Chicago, IL, USA. ${ }^{6}$ Howard Brown Health, Chicago, IL, USA. ${ }^{7}$ Department of Psychiatry, Northwestern University, Feinberg School of Medicine, Chicago, IL, USA.

Received: 3 December 2019 Accepted: 2 January 2020

Published online: 08 January 2020

\section{References}

1. Centers for Diseaese Control and Prevention (CDC). HIV surveillance report, vol. 29; 2017. https:/www.cdc.gov/hiv/library/reports/hiv-surveillance.html; 2018

2. HIV among youth [https://www.cdc.gov/hiv/group/age/youth/index.html].

3. Schulden JD, Song B, Barrosb A, Mares-DelGrasso A, Martind CW, Ramireze R, Smith LC, Wheeler DP, Oster AM, Sullivan PS, et al. Rapid HIV testing in transgender communities by community-based organizations in three cities. Public Health Rep. 2008;123:101-14

4. Chicago Department of Public Health. HIV/STI Surveillance Report, 2013. Chicago: City of Chicago; 2013.

5. Centers for Disease Control and Prevention (CDC). HIV and transgender communities. Atlanta: National Center for HIV/AIDS, Viral Hepatitis, STD, and TB Prevention, Division of HIV/AIDS Prevention; 2019.

6. Hess KL, Chavez PR, Kanny D, DiNenno E, Lansky A, Paz-Bailey G. Binge drinking and risky sexual behavior among HIV-negative and unknown HIV status men who have sex with men, 20 US cities. Drug Alcohol Depend. 2015;147:46-52.

7. Newcomb ME. Moderating effect of age on the association between alcohol use and sexual risk in MSM: evidence for elevated risk among younger MSM. AIDS Behav. 2013;17(5):1746-54.

8. Newcomb ME, Mustanski B. Developmental change in the relationship between alcohol and drug use before sex and sexual risk behavior in young men who have sex with men. AIDS Behav. 2014;18(10):1981-90.

9. Vanable PAMD, Buchbinder SP, et al. Alcohol use and high-risk sexual behavior among men who have sex with men: the effects of consumption level and partner type. Health Psychol. 2004;23(5):525-32.

10. Miller WR, Rollnick S. Motivational interviewing: helping people change. New York: Guilford press; 2013.

11. Rollnick S, Miller WR, Butler C. Motivational interviewing in health care: helping patients change behavior. New York: Guilford press; 2008.

12. Community Prevention Services Task Force. Preventing excessive alcohol consumption: electronic screening and brief intervention (e-SBI). In: Guide to Community Preventive Services. USDHHS; 2012.

13. Collins RL, Parks GA, Marlatt GA. Social determinants of alcohol consumption: the effects of social interaction and model status on the selfadministration of alcohol. J Consult Clin Psychol. 1985;53(2):189-200.

14. Donenberg GR, Emerson E, Bryant FB, Wilson H, Weber-Shifrin E. Understanding AIDS-risk behavior among adolescents in psychiatric care: links to psychopathology and peer relationships. J Am Acad Child Adolesc Psychiatry. 2001;40(6):642-53.

15. Garofalo R, Hotton AL, Kuhns LM, Gratzer B, Mustanski B. Incidence of HIV Infection and Sexually Transmitted Infections and Related Risk Factors Among Very Young Men Who Have Sex With Men. J Acquired Immune Deficiency Syndromes (1999). 2016;72(1):79-86.

16. Garofalo R, Kuhns LM, Reisner SL, Biello K, Mimiaga MJ. Efficacy of an empowerment-based, group-delivered HIV prevention intervention for 
young transgender women: the project LifeSkills randomized clinical trial. JAMA Pediatr. 2018;172(10):916-23.

17. Andresen EM, Malmgren JA, Carter WB, Patrick DL. Screening for depression in well older adults: evaluation of a short form of the CES-D (Center for Epidemiologic Studies Depression Scale). Am J Prev Med. 1994;10(2):77-84.

18. Spitzer RL, Kroenke K, Williams JB, Lowe B. A brief measure for assessing generalized anxiety disorder: the GAD-7. Arch Intern Med. 2006;166(10): 1092-7.

19. Gray MJ, Litz BT, Hsu JL, Lombardo TW. Psychometric properties of the life events checklist. Assessment. 2004;11(4):330-41.

20. Hedeker D, Gibbons RD, Waternaux C. Sample size estimation for longitudinal designs with attrition: comparing time-related contrasts between two groups. J Educ Behav Stat. 1999;24(1):70-93.

21. Cohen J. Statistical power analysis for the behavioral sciences. 2nd ed. Hillsdale: Lawrence Earlbaum Associates; 1988.

22. Cronce JM, Larimer ME. Individual-focused approaches to the prevention of college student drinking. Alcohol Res Health. 2011;34(2):210-21.

\section{Publisher's Note}

Springer Nature remains neutral with regard to jurisdictional claims in published maps and institutional affiliations.

Ready to submit your research? Choose BMC and benefit from:

- fast, convenient online submission

- thorough peer review by experienced researchers in your field

- rapid publication on acceptance

- support for research data, including large and complex data types

- gold Open Access which fosters wider collaboration and increased citations

- maximum visibility for your research: over $100 \mathrm{M}$ website views per year

At BMC, research is always in progress.

Learn more biomedcentral.com/submissions 